\title{
Estado de la investigación en España sobre Inteligencia Emocional en el ámbito educativo
}

\section{Mario Pena Garrido, Elvira Repetto Talavera}

MIDE II (OEDIP), Universidad Nacional de Educación a Distancia, Madrid

\section{España}

Mario Pena Garrido. Facultad de Educación. UNED. c/ Senda del Rey, 7. 28040. Madrid, España. E-mail: mpena@edu.uned.es

(C) Education \& Psychology $\mathrm{I}+\mathrm{D}+\mathrm{i}$ and Editorial EOS (Spain) 


\section{Resumen}

Desde sus orígenes en 1990 el número de investigaciones relativas al concepto de inteligencia emocional (IE) ha experimentado un notable crecimiento. En este artículo se recogen las principales aportaciones de la comunidad científica española en torno a este constructo dentro del ámbito educativo; se exponen los estudios acerca de la naturaleza de la IE y de sus instrumentos de medida, así como la validez de criterio respecto a variables relacionadas con el mundo escolar y universitario; asimismo, se mencionan las principales líneas de trabajo que se llevan a cabo desde la Orientación Educativa, preferentemente en el diseño, aplicación y validación de programas centrados en el desarrollo de las habilidades o competencias de la IE.

Palabras Clave: inteligencia emocional, educación, investigación, bibliografía

Recibido: 03/04/08 Aceptación Provisional: 01/05/08 Aceptación Definitiva: 29/05/08 


\begin{abstract}
Since its origin in 1990, there has been a remarkable rise in the quantity of research relating to the concept of Emotional Intelligence (IQ). This article collects the main contributions from the Spanish scientific community concerning this construct within the field of education; it also discusses research about the nature of EI and instruments for EI measurement, as well as criterion validity with regard to variables relating to the school and university context. In addition, it reviews the main lines of work that are being pursued within Educational Guidance, giving preference to the design, application and validation of programs focused on developing EI skills and competencies.
\end{abstract}

Keywords: emotional intelligence, education, research, references

Received: 04/03/08Ｉnitial Acceptance: 05/01/08ＩFinal Acceptance: 05/29/08 


\section{Introducción}

¿Goza de buena salud la investigación educativa española en torno al concepto de Inteligencia Emocional (IE)? ¿Cuáles son las aportaciones más relevantes dentro de este campo de estudio? Para responder a estas preguntas, se revisa la contribución de la comunidad científica de nuestro país y se exponen las principales líneas de trabajo; en primer lugar, destaca la reciente publicación de cuatro monográficos sobre este tema (Revista Interuniversitaria de Formación del Profesorado, 2005; Psicothema, 2006; Ansiedad y estrés, 2006; Electronic Journal of Research in Educational Psychology, 2008), así como del primer manual en castellano sobre el modelo de habilidad de la IE (2007). En cuanto al número de tesis doctorales españolas que contienen el término IE, aparecen treinta y nueva en la base de datos TESEO, constatándose un aumento en los últimos años. Asimismo destacamos la celebración de eventos científicos de relevancia como el I Congreso Internacional de Inteligencia Emocional en Educación (Las Palmas de Gran Canarias, 2005) y el I Congreso Internacional sobre Inteligencia Emocional (Málaga, 2007), en los que se abordan, entre otros asuntos, la revisión de los modelos conceptuales existentes de la IE, el análisis de los resultados en torno a la evaluación de la IE, y el alcance de la IE en el ámbito aplicado; a todo ello hay que añadir la celebración de cursos y seminarios en las universidades españolas, sin olvidar los programas de doctorado y máster que éstas imparten, cuya temática se centra en la IE.

El objetivo de este artículo es ofrecer una visión de conjunto sobre el estado de la investigación educativa en torno a la inteligencia emocional en España así como destacar la bibliografía más relevante sobre esta cuestión; con este fin, se revisan las aportaciones procedentes de los investigadores del ámbito académico y se articulan sus hallazgos en torno a los cuatro requisitos que Mayer y Salovey $(1997,2007)$ consideran necesarios para identificar una inteligencia dentro de la psicología: a) definirla; b) desarrollar un medio para medirla; c) acreditar su independencia parcial o completa de inteligencias ya conocidas, y d) demostrar que predice algún criterio real; además de estos cuatro criterios no debemos olvidar la importancia del diseño, aplicación y evaluación de programas de intervención en la formación de las habilidades y/o competencias -en función del modelo teórico que se siga- incluidas en la IE, y que están siendo motivo de atención por parte de los investigadores españoles. 
En la actualidad uno de los aspectos más controvertidos en torno a este concepto radica en la naturaleza teórica de la que parten los modelos sobre IE, de modo que podemos distinguir entre modelos de IE basados en el procesamiento de la información emocional centrado en las habilidades emocionales básicas -Mayer y Salovey-, y aquéllos basados en rasgos de personalidad -Goleman y Bar-On- (Fernández-Berrocal y Extremera, 2005; Mestre y Guil, 2003; Mestre, Palmero y Guil, 2004), si bien otros autores, como Fernández-Berrocal y Extremera (2006a y 2006b) señalan la existencia de tres modelos, ya que además de la IE como habilidad, establecen diferencias entre el modelo de Bar-On y el de Goleman; a modo de ejemplo es significativo que el 90\% de los artículos publicados en el monográfico de Ansiedad y estrés (vol. 18, suplemento, 2006) se ajustan al marco teórico propuesto por Mayer y Salovey (Fernández-Berrocal y Extremera, 2006c); asimismo, 9 de los 10 capítulos del $\mathrm{Ma}$ nual de Inteligencia Emocional (Mestre y Fernández-Berrocal, 2007) se fundamentan en los modelos de habilidad quedando un único capítulo para la medida de la IE rasgo. Sin embargo, no es menos cierto, que la mayoría de los estudios publicados hasta el momento utilizan autoinformes, los cuales son más adecuados para medir rasgos de personalidad y nivel de autoeficacia emocional, pero inadecuados para evaluar las habilidades cognitivas implicadas en el procesamiento de la información emocional; esto puede ser debido a las ventajas que ofrecen los cuestionarios frente a las pruebas de habilidad (Extremera y Fernández-Berrocal, 2004) y a la reciente aparición de la adaptación española de la prueba de máximo rendimiento más utilizada en nuestro país como el Mayer-Salovey-Caruso Emotional Intelligence Test (MSCEIT) (Extremera y Fernández-Berrocal, 2002a; Extremera, Fernández-Berrocal y Salovey, 2006).

En España, esta polémica no ha pasado desapercibida (Extremera, 2003; FernándezBerrocal y Extremera, 2005; Mestre, 2003), de ahí que encontremos diferentes estudios en los que se plantea la cuestión acerca de si estamos ante dos modelos que proponen constructos diferentes o bien complementarios; teniendo de fondo la cuestión planteada, observamos que por un lado, Pérez-González, Petrides y Furnham (2007) sostienen que "la operacionalización de la IE como una capacidad cognitiva conduce a un constructo diferente del que se deriva de su operacionalización como un rasgo de personalidad" (p. 97); de esta afirmación se deduce que estamos ante dos constructos diferentes, y por tanto es imprescindible diferenciar claramente entre los métodos de medida para cada una de ellas, de modo que únicamente se utili- 
cen los cuestionarios de rendimiento-máximo para evaluar las habilidades cognitivas, y solamente los autoinformes para medir la IE rasgo (autoeficacia emocional). Por otro lado, se pueden considerar los dos modelos de IE como complementarios (Mestre, 2003) siempre que las medidas utilizadas por ambos modelos no correlacionen significativamente entre sí por encima de un valor de $\mathrm{r}=0.3$-si se tratan de escalas-, a la vez que cada medida aporte una varianza independiente explicada con respecto a algún criterio establecido (Mestre, Guil y Gil-Olarte 2004); aunque los resultados de esta investigación muestran ausencia de relaciones significativas entre las subescalas del MSCEIT y del Cuestionario de Inteligencia Emocional como rasgo para escolares - CIE - (Mestre, 2003) - por lo que, en principio dichos instrumentos estarían midiendo conceptos teóricamente diferentes -, sin embargo, "no queda claro si existen diferentes formas de explicar cómo las personas manejan eficazmente las emociones, por lo que se necesitaría mayor número de investigaciones con varias medidas validadas de IE (como rasgo y como habilidades) respecto a varios criterios adaptativos que nos permitieran si son válidas ambas formas de entender la IE” (Mestre, Guil y Gil-Olarte, 2004).

\section{Instrumentos de medida}

En cuanto a los instrumentos de medida de la IE, una rigurosa y extensa revisión ha sido llevada a cabo por Extremera y Fernández-Berrocal (2007), así como por Extremera, Fernández-Berrocal, Mestre y Guil (2004) y Pérez-González, Petrides y Furnham (2005, 2007). Los tres métodos principales en la medición de la IE son los autoinformes, las pruebas de ejecución y la evaluación 360 grados (Extremera y Fernández-Berrocal, 2003a); en este sentido, uno de los campos más fructíferos radica en la adaptación y validación al castellano de cuestionarios de inteligencia emocional - tanto desde la perspectiva de la IE habilidad como de la IE rasgo -, así como la constatación de su validez predictiva; llama la atención la aparente contradicción que puede suponer el uso generalizado de autoinformes basados en el modelo teórico de Mayer y Salovey, como ocurre con la Trait Meta Mood Scale -TMMS(Extremera y Fernández-Berrocal, 2005a); sin embargo existen evidencias de que combinados junto con una prueba de habilidad como es el MSCEIT, pueden aportar una mayor varianza respecto a una determinada variable criterio, tal y como ocurre con el cociente intelectual y la motivación respecto al rendimiento académico (Mestre y Guil, 2006); de hecho, las pruebas de habilidad de la IE nos revelan si un alumno tiene desarrolladas o no estas habilidades, pero no nos dice nada sobre si ese mismo alumno las pone en práctica en su vida diaria, aspecto que sí es destacado en los autoinformes ya que éstos nos informan del grado de autoeficacia emocional de un sujeto. Por otro lado, el MSCEIT se ha revelado como la prueba más ade- 
cuada hasta el momento para medir las habilidades cognitivas de la IE, sin obviar los puntos débiles que presenta, como por ejemplo la ausencia de un criterio objetivo para determinar en qué consiste una respuesta emocional correcta; el hecho de que este instrumento está relacionado con medidas de inteligencia verbal, por lo que los sujetos más inteligentes también obtienen una mejor puntuación en el MSCEIT (Mestre, Guil y Gil-Olarte, 2004); o el escaso poder predictor de la rama percepción; llama la atención el escaso poder predictor de la rama percepción respecto a una variable como el número de veces que es elegido como preferido/a, mientras que sí obtuvo relaciones positivas y significativas con las otras tres ramas del MSCEIT; asimismo, un estudio posterior (Mestre, Guil y Mestre, 2005), en contra de lo esperado, confirma la ausencia de relación significativa entre la rama de "percepción de las emociones" del MSCEIT con otra variable objetiva como el reconocimiento de las emociones medida a través del DANVA (Diagnostic Analisis of Nonverbal Accuracy), por lo que los autores de esta investigación sugieren utilizar una prueba alternativa a esta rama debido a la escasez de resultados sobre validez predictiva que está teniendo, como ya se viene haciendo en el ámbito internacional.

Finalmente, la tercera vía para medir la IE es la evaluación $360^{\circ}$ o feedback $360^{\circ}$ (Extremera y Fernández-Berrocal, 2003a), la cual a pesar de ser la menos utilizada, se está revelando como un recurso adecuado en el proceso de desarrollo y evaluación de los programas de educación emocional (Bisquerra, Martínez, Obiols y Pérez, 2006); su utilización nos permite comparar las valoraciones de tres categorías diferentes de informantes: el propio sujeto, el profesorado y el alumnado; asimismo, facilita el contraste entre las valoraciones de un grupo de profesores que imparten docencia sobre el mismo alumnado, y nos permite comparar las autoevaluaciones de un alumno con las del profesorado.

\section{Poblaciones utilizadas en la investigación}

Otro asunto que ha ocupado a los estudiosos, y que no es baladí, hace referencia a la necesidad de distinguir entre las investigaciones cuya muestra elegida son alumnos universitarios de aquellas otras en las que los sujetos se enmarcan en la educación secundaria; la importancia radica en el hecho de que, como señalan Mestre y Guil (2006), "las muestras heterogéneas como el alumnado de secundaria, nos permiten acceder a un mayor abanico de sujetos con diferente nivel de desarrollo de las habilidades implícitas en la IE, ya que la elección de muestras universitarias no nos permite ver los efectos de un mal desarrollo de la IE, puesto que los participantes han ido superando diversos filtros escolares y de éxito para poder llegar 
a estos niveles educativos, proceso al que la IE probablemente contribuye". Por ejemplo, Mestre y Guil (2003) llaman la atención sobre el hecho de que con muestras heterogéneas como las de secundaria - se constatan las diferencias en IE a favor de las mujeres frente a los hombres, mientras que en muestras homogéneas - universitarias - esto no ocurre tan claramente. Por tanto sería deseable, en la medida de lo posible, fomentar el empleo de muestras escolarizadas en Secundaria frente a las universitarias.

\section{Diferencias de género}

Las diferencias en IE desde la perspectiva de género es otro de los aspectos resaltados en la investigación (Mestre, Guil y Lim, 2004) y en la que todos coinciden en señalar la presencia de rasgos o habilidades más desarrollados en función del hecho de ser hombre o mujer, en donde cada género presenta un perfil personal de puntos fuertes y débiles (Caballero, 2004). Estas diferencias de género sugieren tratar los estudios de validez predictiva de la IE por separado -hombres y mujeres - para evitar efectos de regresión a la media (Mestre y Guil, 2006); en este sentido, es muy interesante la aportación de Mestre, Núñez-Vázquez y Guil (2007), en el que se presentan los aspectos diferenciales, en cuanto al género, de las cuatro habilidades del modelo de Mayer y Salovey; paralelamente, es significativo el hecho de que, dependiendo del tipo de medida utilizada - autoinforme o prueba de habilidad - las diferencias observadas sean distintas en función del género (Extremera, Fernández-Berrocal y Salovey, 2006; Fernández-Berrocal, Extremera y Ramos, 2004).

\section{Validez discriminante}

El último aspecto de este apartado hace referencia a la independencia de la IE respecto a otros conceptos ya conocidos; a juicio de Mestre y Guil (2006) y Mestre, Comunian y Comunian (2007), quizás sea éste el aspecto más descuidado por parte de los autores originales del constructo. Para saber si "estamos ante un constructo de inteligencia, o por el contrario, ante uno de personalidad, o podemos estar ante un constructo realmente novedoso aunque relacionado con ambos términos de una manera no implícita" es necesario verificar que los índices de correlación entre ellos no están por encima de 0.6 (Mestre, Guil y Mestre, 2005). Para comprobar que el concepto de IE es novedoso respecto a otros ya existentes, también se ha realizado una correlación entre las medidas de la IE y las del AECS (Actitudes y estrategias cognitivas sociales) (Mestre, Guil y Gil-Olarte, 2004). Por otra parte, la aparición de correlaciones prácticamente nulas, ninguna de ellas significativa, entre el cociente intelectual que define la inteligencia psicométrica tradicional y los diferentes aspectos de la inteligencia 
emocional evaluados en el estudio, indica la independencia de ambos tipos de inteligencia, en línea con los resultados de otras investigaciones (Extremera y Fernández-Berrocal, 2004; Pérez y Castejón, 2007).

\section{Predicción de criterios objetivos y/o subjetivos}

En este apartado, se exponen las investigaciones en torno a la validez predictiva de la IE dentro del ámbito educativo; es decir, nos planteamos si este constructo es útil en la formación de los estudiantes y qué ventajas se derivan de ello; con este propósito, las variables más estudiadas se agrupan en torno al concepto de adaptación escolar, en el cual se incluyen variables objetivas (rendimiento académico, número de veces que ha sido elegido como buen compañero, partes de conflictos de los profesores sobre los alumnos) y variables subjetivas (percepción de clima en el aula, autoevaluación); asimismo consideramos la relación entre la IE y el consumo de tabaco y alcohol debido a la importancia y al auge que está teniendo entre los adolescentes; finalmente se menciona la utilización de la madurez vocacional como otras de las variables criterio a tener en cuenta.

El rendimiento académico ha sido una de las variables más utilizadas para medir el poder predictor de la IE, hallándose relaciones positivas y significativas con tres de las cuatro escalas del MSCEIT -con la excepción de la rama percepción- así como con su puntuación total, y con la subescala de autoeficacia del CIE (Mestre, Guil y Gil-Olarte, 2004); en esta misma línea, Gil-Olarte, Palomera y Brackett (2006) encontraron correlaciones positivas, estadísticamente significativas, entre las puntuaciones del MSCEIT y las calificaciones académicas, incluso después de controlar los factores de personalidad e inteligencia. Gil-Olarte, Guil, Mestre, y Nuñez (2005) extraen la misma conclusión ya que hallaron evidencias de correlaciones significativas positivas entre IE y rendimiento académico con estudiantes de secundaria. Si nos centramos en las investigaciones que recurren a autoinformes que evalúan la inteligencia emocional percibida, los resultados con muestra universitaria (Pérez y Castejón, 2006 y 2007) señalan la existencia de relaciones moderadas, aunque significativas, de los factores emocionales con el rendimiento académico en estos estudiantes, incluso cuando se controla el efecto de la inteligencia psicométrica tradicional.

De todo lo expuesto en este apartado hasta el momento, se concluye que la IE se considera un factor explicativo del rendimiento académico, pero como una relación directa, sin considerar los mecanismos que subyacen; una de las aportaciones más valiosas de Extremera 
y Fernández-Berrocal (2003b) dentro del ámbito escolar, con una muestra de estudiantes de $3^{\circ}$ y $4^{\circ}$ de E.S.O., incide en el efector mediador que una buena salud mental ejerce sobre el rendimiento medio escolar de los estudiantes; es decir, aquellos alumnos que informaron de un mayor nivel de inteligencia emocional percibida, medida a través del TMMS-24, mostraron menor sintomatología depresiva y ansiosa y menor tendencia a tener pensamientos intrusivos; y a su vez, los alumnos con un nivel inferior de ajuste psicológico (mayor nivel de depresión) alcanzaron un menor rendimiento académico al finalizar el trimestre. Posteriores estudios (Durán, Extremera, Rey, Fernández-Berrocal y Montalbán, 2006; Extremera, Durán y Rey, 2007a) plantean la hipótesis de que los alumnos con mayores niveles de IE muestran a lo largo del curso menores sentimientos de agotamiento, cinismo y estrés y mayores actitudes positivas hacia sus tareas (mayores niveles de vigor, dedicación y absorción), efectos positivos que podrían a su vez explicar parcialmente la relación positiva y significativa corroborada en los estudios longitudinales que vinculan IE y rendimiento escolar.

La adaptación socio-escolar, entendida como frecuencia de comportamientos conflictivos, el rendimiento académico y el número de veces que ha sido elegido como buen compañero ha sido estudiada principalmente por el grupo de la Universidad de Cádiz (Guil y GilOlarte, 2007; Guil, Gil-Olarte, Mestre, y Nuñez, 2005, 2006; Mestre y Guil, 2006; Mestre, Guil y Gil-Olarte, 2004; Mestre, Guil, Lopes, Salovey y Gil-Olarte, 2006), a los que hay que añadir las investigaciones realizadas por el grupo de investigación de la Universidad de Málaga (Extremera y Fernández-Berrocal, 2003b). Si nos centramos en la conflictividad en el aula, se aprecia que los sujetos que mejor usan, comprenden y manejan sus emociones -MSCEIT- son aquellos que obtienen menor número de "partes" por faltas de indisciplina y por agresión, siendo menos hostiles en clase (Mestre, Guil y Gil-Olarte, 2004). De ahí que se proponga la IE como factor protector de conductas problema como la violencia, la impulsividad y el desajuste emocional (Extremera y Fernández-Berrocal, 2002b; Mestre, Guil y Segovia, 2007). En un estudio con adolescentes entre 13 y 17 años (Mestre et al., 2005), la IE no muestra relaciones significativas respecto al número de veces que un alumno es elegido como preferido por sus compañeros, ni tampoco con la autovaloración sobre el funcionamiento personal y social dentro del aula; asimismo, Mestre, Guil y Gil-Olarte (2004) hallaron que a pesar de que la variable número de veces que es elegido como preferido/a se relacionó con todas las medidas del MSCEIT salvo percibir emociones, sin embargo en el análisis de regresión sólo la rama manejo de emociones mostró cierto grado de predicción, por lo que se deduce que la IE no parece predecir una mejor aceptación social o valoración personal de los alumnos; la explica- 
ción la encontramos en Mestre et al. (2005) quienes afirman que la IE no predice el ajuste personal debido a que se utilizan medidas subjetivas en vez de hacerlo con criterios objetivos, como el rendimiento académico, en los que sí se ha verificado su poder predictor.

Asimismo, la IE se asocia con la disminución de conductas de riesgo para la salud entre las que se incluyen el consumo de tabaco y alcohol (Ruiz-Aranda, Fernández-Berrocal, Cabello y Extremera, 2006; Senra, Pérez-González y Manzano 2007); en concreto, "las personas con una falta de habilidades emocionales pueden recurrir al consumo de drogas como una forma externa de autorregulación para disminuir, ocultar o ignorar sus estados emocionales negativos o para generar estados emocionales más agradables" (Fernández-Berrocal y Extremera, 2007, pp.182).

Destacamos por su novedad las aportaciones de Vila y Pérez-González (2007) acerca de la IE rasgo y la madurez vocacional (planificación de la carrera, exploración de la carrera, toma de decisiones e información sobre el mundo laboral) medida a través del Carrer Development Inventory - CDI - de acuerdo con los cuales se hallaron coeficientes moderados pero significativos estre las dos variables, más concretamente entre la IE rasgo y el componente actitudinal de la madurez vocacional, mientras que no ocurre lo mismo entre la IE rasgo y las escalas de carácter cognitivo del CDI. Sin embargo no hay que olvidar que antes de generalizar el uso de la IE en el asesoramiento vocacional y profesional se precisa establecer mejor el constructo y sus medidas antes de conducir intervenciones, por ejemplo, en la Psicología Vocacional, ya que su relación con la conducta vocacional, con la elección vocacional o con el rendimiento en el trabajo no está rigurosamente establecida (Valls, 2007).

Finalmente, otras variables que han sido motivo de atención por parte de los investigadores y que se han relacionado con la IE son: Estrés, engagement y burnout (Extremera, Durán y Rey, 2007a), ansiedad y estrés en adolescentes (Fernández-Berrocal, Alcaide, Extremera, y Pizarro, 2006; Repetto, Pena y Lozano, 2006), optimismo y pesimismo (Extremera, Durán y Rey, 2007b), salud física y mental (Extremera y Fernández-Berrocal, 2006), satisfaccion personal (Extremera y Fernández-Berrocal, 2005b), empatía (Fernández y Barraca, 2005), calidad de vida (Martínez de Antoñana, Pulido, Berrios, Augusto, Luque y López, 2004) y apoyo a la integración escolar (Lucas, 2005). 


\section{Orientación Educativa y programas de intervención}

La Orientación Educativa en España concede un papel relevante a la formación en competencias socio-emocionales de los alumnos (Bisquerra, 2002, 2004a, 2004b; Bisquerra y Pérez, 2007; Repetto, 2003), poniendo el acento en la necesidad de diseñar, aplicar y evaluar programas de intervención sobre las habilidades y/o competencias de la IE (Álvarez, 2001; Bisquerra, 2004a) consciente de que el aprendizaje de estas competencias no depende tanto de la instrucción verbal como de la práctica y el entrenamiento (Fernández-Berrocal y Extremera, 2002); aunque son cada vez más numerosas las propuestas de programas de educación emocional en educación infantil, primaria y secundaria (Repetto, Pena, Mudarra y Uribarri, 2007), sin embargo, se aprecia un déficit en cuanto a la validación de los mismos, siendo difícil encontrar estudios publicados sobre su evaluación y eficacia (Guil y Gil-Olarte, 2007; Muñoz de Morales y Bisquerra, 2006; Repetto, Pena y Lozano, 2007); además, sería deseable un estudio actualizado sobre los diversos programas en IE de nuestro país, analizando sus características principales (modelo teórico en el que se fundamentan, objetivos específicos, destinatarios, competencias o habilidades entrenadas, metodología, temporalización) y los resultados empíricos de su aplicación, si los hubiere; un primer intento de este acercamiento ha sido llevado a cabo por Vallés y Vallés $(2000,2003)$. Un paso previo en la aplicación de programas educativos en la escuela para el desarrollo de la IE consiste en la necesidad de formar a los profesores que van a impartirlos (Bisquerra, 2005; Obiols, 2005; Palomera, Gil-Olarte y Brackett, 2006), pues los datos indican que los docentes están muy sensibilizados ante la necesidad de incluir la educación emocional en las aulas pero que no han recibido la formación e instrucción necesaria para llevarla a cabo con éxito (Abarca, Marzo y Sala, 2002; Hué, 2007). La iniciativa de Muñoz de Morales (2005) propone el desarrollo de competencias emocionales en los docentes a través del programa P.E.C.E.R.A. (Programa Educativo de Conciencia Emocional, Regulación y Afrontamiento) como paso previo para una adecuada intervención con su alumnado; a esto hay que añadir el efecto protector de la IE frente al burnout que sufren muchos profesores como consecuencia del estrés al que se ven sometidos en su labor profesional (Extremera, Fernández-Berrocal y Durán, 2003). Todo esto adquiere una mayor importancia cuando se trata de los profesores-tutores ya que "son considerados como un factor determinante para promover la formación en competencias socio-emocionales de los estudiantes y recién titulados” (Repetto y Pérez-González, 2007, pp. 105).

Lo expuesto hasta ahora no excluye otras iniciativas que han surgido dentro de las áreas curriculares como la literatura (Gaya y Sánchez-Doreste, 2006; Pena, 2007) Tampoco han 
faltado las voces que desean incluir en el currículo escolar una materia o asignatura sobre educación emocional (Bisquerra, 2000; Vallés, 2008), de modo que la formación de los alumnos en este terreno no dependa exclusivamente de la sensibilización del equipo educativo. Finalmente la propuesta de actividades para el desarrollo de las habilidades de la IE en el ámbito escolar (Extremera y Fernández-Berrocal, 2002c; Fernández-Berrocal y Ramos, 2004; Gallego y Gallego, 2004; Vallés, 2008) ha sido motivo de atención y reflexión por parte de la comunidad científica española.

\section{Conclusiones}

Las aportaciones de los investigadores españoles dentro del campo de la educación se articulan en torno a la clarificación del concepto y de los modelos de IE - rasgo y habilidad así como de sus instrumentos de medida; además, se ha tratado de diferenciar esta inteligencia respecto a otros constructos relacionados como el cociente intelectual y la personalidad, sin olvidar la utilidad de la IE para el mundo educativo - validez de criterio -; de acuerdo a los resultados obtenidos hasta el momento se puede afirmar que la IE parece aportar cierto grado explicativo en el ajuste socioescolar del alumnado, especialmente en lo que se refiere a rendimiento académico y conductas disruptivas en el aula, así como respecto a otras variables como el estrés y la ansiedad de los alumnos, el consumo de tabaco y alcohol, el optimismo y la madurez vocacional. Además, se constata la necesidad de diseñar, aplicar y evaluar programas de intervención centrados en las habilidades o competencias de la IE con el objetivo de fomentar el crecimiento personal y facilitar el acceso al mundo laboral. Por tanto, podemos concluir, que la investigación española en torno a la IE goza de buena salud, aunque ello no exima de los retos que han de afrontarse en el futuro más inmediato como Salovey (2007) señala en el prólogo del primer Manual de Inteligencia Emocional en castellano (Mestre y Fernández-Berrocal, 2007): ¿qué relación existe entre los dos modelos de IE? ¿Cómo desarrollar medidas de la IE menos dependientes del conocimiento y más de las habilidades cognitivas? ¿Existen dimensiones de la IE no contempladas en el modelo de habilidad? ¿Cuál es el rol de la cultura en la IE? ¿Qué aportan los acercamientos neurocientíficos a la IE? 


\section{Referencias}

Abarca, M., Marzo, L. y Sala, J. (2002). La educación emocional en la práctica educativa de primaria. Bordón, 54, 4, 505-518.

Álvarez, M. (2001). Diseño y evaluación de programas de educación emocional. Barcelona: Ciss-Praxis.

Bisquerra, R. (2000). Educación emocional y bienestar. Barcelona: Praxis.

Bisquerra, R. (2002). La competencia emocional. En M. Álvarez y R. Bisquerra, Manual de orientación y tutoría (pp.69-83). Barcelona: Praxis.

Bisquerra, R. (2004a). Diseño, aplicación y evaluación de programas de educación emocional. En M.J. Iglesias (ed.): El reto de la educación emocional en nuestra sociedad, (pp. 121-161). A Coruña, Universidad de Coruña.

Bisquerra, R. (2004b). Orientación y competencias emocionales. En AA.VV., Actas de la Conferencia Internacional Orientación, Inclusión Social y Desarrollo de la Carrera, pp.198-210. Universidad de Coruña.

Bisquerra, R. (2005). La educación emocional en la formación del profesorado. Revista Interuniversitaria de Formación del Profesorado, 19 (3), 95-114.

Bisquerra, R., Martínez, F., Obiols, M. y Pérez, N. (2006). Evaluación de 360: Una aplicación a la educación emocional. Revista de Investigación Educativa (RIE), 24 (1), 187203.

Bisquerra, R. y Pérez, N. (2007). Las competencias emocionales. Educación XXI, 10, 61-82.

Caballero, A. (2004). Cómo enfocar la educación emocional dentro del aula desde la perspectiva de género. En AA.VV., Actas de la Conferencia Internacional Orientación, Inclusión Social y Desarrollo de la Carrera, pp.546-550. Universidad de Coruña.

Durán, A., Extremera, N., Rey, L., Fernández-Berrocal, P. y Montalbán, F.M. (2006). Predicting academic burnout and engagement in educational settings: Assessing the incremental validity of perceived emotional intelligence beyond perceived stress and general self-efficacy. Psicothema, 18, 158-164.

Extremera, N. (2003). El modelo de inteligencia emocional de Mayer y Salovey y su validez predictiva en muestras españolas. Tesis doctoral. Universidad de Málaga.

Extremera, N., Durán, A. y Rey, L. (2007a). Inteligencia emocional y su relación con los niveles de burnout, engagement y estrés en estudiantes universitarios. Revista de Educación, 342, 239-256 
Extremera, N., Durán, A. y Rey, L. (2007b). Perceived emotional intelligence and dispositional optimism-pessimism: Analyzing their role in predicting psychological adjustment among adolescents. Personality and Individual Differences, 42, 1069-1079.

Extremera, N. y Fernández-Berrocal, P. (2002a). Adaptación al castellano del Mayer-SaloveyCaruso Emotional Intelligence Test (MSCEIT): User's Manual. Toronto: Multi-Health Systems, Inc.

Extremera, N. y Fernández-Berrocal, P. (2002b). La inteligencia emocional en el aula como factor protector de conductas problema: violencia, impulsividad y desajuste emocional. En F. A. Muñoz, B. Molina y F. Jiménez (Eds.) Actas I Congreso Hispanoamericano de Educación y Cultura de Paz (pp. 599-605). Editorial Universidad de Granada: Granada.

Extremera, N. y Fernández-Berrocal, P. (2002c). Educando emociones. La educación de la inteligencia emocional en la escuela y la familia. En Fernández Berrocal, P. y Ramos Díaz, N. Corazones inteligentes. Barcelona: Kairos.

Extremera, N. y Fernández-Berrocal, P. (2003a). La inteligencia emocional: Métodos de evaluación en el aula. Revista Iberoamericana de Educación, 30, 1-12.

Extremera, N. y Fernández-Berrocal, P. (2003b). La inteligencia emocional en el contexto educativo: Hallazgos científicos de sus efectos en el aula. Revista de Educación, 332, 97-116.

Extremera, N. y Fernández-Berrocal, P. (2004). El uso de las medidas de habilidad en el ámbito de la inteligencia emocional. Ventajas e inconvenientes con respecto a las medidas de auto-informe. Boletín de Psicología, 80, 59-77.

Extremera, N. y Fernández-Berrocal, P. (2005a). Inteligencia emocional percibida y diferencias individuales en el metaconocimiento de los estados emocionales: una revisión de los estudios con el Trait Meta-Mood Scale. Ansiedad y estrés, 12, 191-205.

Extremera, N. y Fernández-Berrocal, P. (2005b). Perceived emotional intelligence and life satisfaction: Predictive and incremental validity using the Trait Meta-Mood Scale. Personality and Individual Differences, 39, 937-948.

Extremera, N. y Fernández-Berrocal, P (2006). Emotional intelligence as predictor of mental, social and physical health in university students. The Spanish Journal of Psychology, 9, 45-51.

Extremera, N. y Fernández-Berrocal, P. (2007). Una guía práctica de los instrumentos actuales de evaluación de la inteligencia emocional. En Mestre, J.M. y Fernández-Berrocal, P. (Eds): Manual de Inteligencia emocional, 99-122. Madrid, Ed. Pirámide. 
Extremera, N. y Fernández-Berrocal, P. y Durán, A. (2003). Inteligencia emocional y burnout en profesores. Encuentros en Psicología Social, 1, 260-265.

Extremera, N., Fernández-Berrocal, P., Mestre, J.M. y Guil, R. (2004). Medidas de evaluación de la inteligencia emocional. Revista Latinoamericana de Psicología, 36 (2), 209-228.

Extremera, N., Fernández-Berrocal, P. y Salovey, P. (2006). Spanish version of the MayerSalovey-Caruso Emotional Intelligence Test (MSCEIT). version 2.0: reliabilities, age and gender differences, Psicothema, 18, suplemento, 42-48.

Fernández, A. y Barraca, J. (2005). Inteligencia emocional, empatía y competencia social. Una investigación empírica con estudiantes universitarios. En J. Romay y R. García (Eds.), Psicología social y problemas sociales. Psicología Ambiental, Comunitaria y Educación (pp. 335-342). Madrid: Biblioteca Nueva.

Fernández-Berrocal, P., Alcaide, R., Extremera, N. y Pizarro, D.A. (2006). The role of emotional intelligence in anxiety and depression among adolescents. Individual Differences Research, 4, 16-27.

Fernández-Berrocal, P. y Extremera, N. (2002). La inteligencia emocional como una habilidad esencial en la escuela. Revista Iberoamericana de Educación, 29, 1-6.

Fernández-Berrocal, P. y Extremera, N. (2005). La inteligencia emocional y la educación de las emociones desde el Modelo de Mayer y Salovey. Revista Interuniversitaria de Formación del Profesorado, 19, 63-93.

Fernández-Berrocal, P. y Extremera, N. (2006a). Special issue on emotional intelligence: An overview. Psicothema, 18, suplemento, 1-6.

Fernández-Berrocal, P. y Extremera, N. (2006b). Emotional intelligence: A theoretical and empirical overview of its first 15 years of history. Psicothema, 18, 7-12.

Fernández-Berrocal, P. y Extremera, N. (2006c). La investigación de la inteligencia emocional en España. Ansiedad y Estrés, 12 (2-3), 139-153.

Fernández-Berrocal, P. y Extremera, N. (2007). Inteligencia emocional y salud. En Mestre, J.M. y Fernández-Berrocal, P. (Eds): Manual de Inteligencia emocional, (pp. 173187). Madrid, Ed. Pirámide.

Fernández-Berrocal, P., Extremera, N. y Ramos, N. (2004). Validity and reliability of the Spanish modified version of the Trait Meta-Mood Scale. Psychological Reports, 94, 751-755.

Fernández-Berrocal, P. y Ramos, N. (2004). Desarrolla tu inteligencia emocional. Barcelona: Kairos. 
Gaya, J. y Sánchez-Doreste, J. (2006). El club de los poetas muertos, un referente para el desarrollo de la Inteligencia Emocional, Aula de innovación educativa, 156, 18-23.

Gallego, D.J. y Gallego, M.J. (2004). Educar la inteligencia emocional en el aula. Madrid: PPC.

Gil-Olarte, P., Guil, R., Mestre, J.M., y Nuñez, I. (2005). La Inteligencia emocional como variable predictora del rendimiento académico. En J. Romay y R. García (Eds.), Psicología social y problemas sociales. Psicología Ambiental, Comunitaria y Educación (pp. 351357). Madrid: Biblioteca Nueva.

Gil-Olarte, P., Palomera, R. y Brackett, M. (2006). Relating emotional intelligence to social competence and academic achievement in high school students. Psicothema, 18, supl., 118-123.

Guil, R. y Gil-Olarte, P. (2007). Inteligencia emocional y educación: desarrollo de competencias socio-emocionales. En Mestre, J.M. y Fernández-Berrocal, P. (Eds): Manual de Inteligencia emocional, 189-215, Madrid, Ed. Pirámide.

Guil, R., Gil-Olarte, P., Mestre, J.M., y Nuñez, I. (2005). Inteligencia emocional y adaptación socioescolar. En J. Romay y R. García (Eds.), Psicología social y problemas sociales. Psicología Ambiental, Comunitaria y Educación (pp. 359-366). Madrid: Biblioteca Nueva.

Guil, R., Gil-Olarte, P., Mestre, J.M., y Nuñez I. (2006). Inteligencia emocional y adaptación socioescolar. Revista Electrónica de Motivación y Emoción, IX (22). Recuperado el 29 de enero de 2008 de http://reme.uji.es/reme/numero22/index-sp.html.

Hué, C. (2007). Bienestar docente y pensamiento emocional. Wolters Kluvert.

Lucas, J.M. (2005). Desarrollo de la inteligencia Emocional como apoyo a la integración escolar, Campo abierto: Revista de Educación, 28, 81-96.

Martínez de Antoñana, R., Pulido, M., Berrios, M.P., Augusto, J.M., Luque, P.J. y López, E. (2004). Inteligencia emocional percibida y calidad de vida en estudiantes universitarios. Revista de Psicología Social Aplicada, 14 (2), 61-78.

Mayer, J.D., y Salovey, P. (1997). What is emotional intelligence? En P. Salovey y D.J. Sluyter (Eds), Emotional development and emotional intelligence, (pp. 3-31). Nueva York: Basic Book.

Mayer, J.D. y Salovey, P. (2007). ¿Qué es inteligencia emocional? En Mestre, J.M. y Fernández-Berrocal, P. (Eds): Manual de Inteligencia emocional, (pp. 25-45), Madrid, Ed. Pirámide. 
Mestre, J.M. (2003). Validación empírica de una prueba para medir la inteligencia emocional en una muestra de estudiantes de la Bahía de Cádiz. Tesis doctoral. Universidad de Cádiz.

Mestre, J.M., Comunian, A.L. y Comunian, M.L. (2007). Inteligencia emocional: una revisión a sus primeros quince años y un acercamiento conceptual desde los procesos psicológicos. En Mestre, J.M. y Fernández-Berrocal, P. (Eds): Manual de Inteligencia emocional, (pp. 47-68). Madrid, Ed. Pirámide.

Mestre, J.M. y Fernández-Berrocal, P. (2007). Manual de inteligencia emocional. Madrid, Pirámide.

Mestre, J.M. y Guil, R. (2003). Inteligencia emocional. En Fernández-Abascal, M.P. Jiménez y M.D. Martín, (Eds.), Emoción y Motivación. La adaptación humana. (pp. 397-425). Madrid: Centro de Estudios Ramón Areces.

Mestre, J.M. y Guil, R. (2006). Medidas de ejecución versus medidas de autoinformes de inteligencia emocional. Ansiedad y Estrés, 12(2-3), 413-425.

Mestre, J.M., Guil, R. y Gil-Olarte, P. (2004). Inteligencia emocional: algunas respuestas empíricas y su papel en la adaptación escolar en una muestra de alumnos de secundaria. Revista Electrónica de Motivación y Emoción, VI (16), recuperado el 29 de enero de 2008 de http://reme.uji.es/reme/numerol6/index-sp.html.

Mestre, J.M., Guil, R. y Lim, N. (2004). Inteligencia emocional: ¿a qué pueden deberse las diferencias respecto a la variable género? En E. Barberá, L. Mayor, M. Chóliz, E. Cantón, E. Carbonell, C. Candela y C. Gómez (Eds.), Motivos, emociones y procesos representacionales: de la teoría a la práctica (pp. 313-324). Valencia. Fundación Universidad-Empresa de Valencia (ADEIT).

Mestre, J.M., Guil, R., Lopes, P., Salovey, P. y Gil-Olarte, P. (2006). Emotional Intelligence and social and academic adaptation to school. Psicothema, 18, 112-117.

Mestre, J.M., Guil, R. y Mestre, R. (2005). Inteligencia emocional: resultados preliminares sobre su naturaleza y capacidad predictiva a partir de un estudio correlacional en muestras de estudiantes de secundaria. Revista Española de Orientación y Psicopedagogía, 16, 2(2), 269-281.

Mestre, J.M., Guil, R. y Segovia, F. (2007). Inteligencia emocional: un valor preventivo de la conflictividad en el aula. I Congreso Internacional de Inteligencia Emocional.

Mestre, J.M., Nuñez-Vázquez, I. y Guil, R. (2007). Aspectos psicoevolutivos, psicosociales y diferenciales de la inteligencia emocional. En Mestre, J.M. y Fernández-Berrocal, P. (Eds): Manual de Inteligencia emocional (pp. 153-172). Madrid, Ed. Pirámide. 
Mestre, J.M., Palmero, F. y Guil, R. (2004). Inteligencia emocional: una explicación integradora desde los procesos psicológicos básicos. En J.M. Mestre y F. Palmero (coords.), Procesos psicológicos básicos: una guía académica para los estudios en Psicopedagogía, Psicología y Pedagogía. (pp. 249-280). Madrid: McGraw-Hill.

Muñoz de Morales, M. (2005). Prevención del estrés psicosocial del profesorado mediante el desarrollo de competencias emocionales: el programa P.E.C.E.R.A. Revista Interuniversitaria de Formación del Profesorado, 19 (3), 115-136.

Muñoz de Morales, M. y Bisquerra, R. (2006). Evaluación de un programa de educación emocional para la prevención del estrés psicosocial en el contexto del aula. Ansiedady estrés, 12 (2-3), 401-412.

Obiols, M. (2005). Diseño, desarrollo y evaluación de un programa de educación emocional en un centro educativo. Revista interuniversitaria de Formación del Profesorado, 19 (3), 137-152.

Palomera, R., Gil-Olarte P. y Brackett, M.A. (2006). ¿Se perciben con inteligencia emocional los docentes? Posibles consecuencias sobre la calidad educativa. Revista de Educación, 341, 687-703.

Pena, M. (2007). La Orientación y Formación en competencias socio-emocionales en función del género, el curso académico y la dedicación a la lectura. En F. Etxeberría, L. Sarasola, J.F. Lukas, J. Etxeberría y A. Martxueta (coords.), XIII Congreso Nacional de Modelos de Investigación Educativa (pp. 712-717). Donostia: Erein/AIDIPE (Libro en CD-Rom; ISBN: 978-84-9746-382-9).

Pérez, N. y Castejón, J.L. (2006). Relaciones entre la inteligencia emocional y el cociente intelectual con el rendimiento académico en estudiantes universitarios. Revista Electrónica de Motivación y Emoción, IX, 22; recuperado el 29 de enero de 2008 de http://reme.uji.es/reme/numerol6/index-sp.html.

Pérez, N. y Castejón, J.L. (2007). La inteligencia emocional como predictor del rendimiento académico en estudiantes universitarios. Ansiedad y estrés, 13 (1), 119-129.

Pérez-González, J.C., Petrides, K.V. y Furnham, A. (2005). Measuring trait emotional intelligence. En R. Schulze y R. Roberts (Eds.), International Handbook of Emotional Intelligence (pp. 181-201). Cambridge, MA: Hogrefe \& Huber.

Pérez-González, J.C., Petrides, K.V. y Furnham, A. (2007). La medida de la inteligencia emocional rasgo. En Mestre, J.M. y Fernández-Berrocal, P. (Eds): Manual de Inteligencia emocional, (pp. 81-98). Madrid, Ed. Pirámide. 
Repetto, E. (2003). La competencia emocional e intervenciones para su desarrollo. En E.Repetto (dir): Modelos de Orientación e Intervención Psicopedagógica, vol. II (p. 454-482). Madrid: UNED.

Repetto, E., Pena, M. y Lozano, S. (2006). Adaptación y validación española del Emotional Intelligence Inventory (EII): estudio piloto. Ansiedad y estrés, 12 (2-3), 181-189.

Repetto, E., Pena, M. y Lozano, S. (2007). El programa de competencias socio-emocionales (POCOSE). XXI Revista de Educación, 9, 35-41.

Repetto, E., Pena, M., Mudarra, M.J. y Uribarri, M. (2007). Guidance in the area of socioemotional competencies for secondary students in multicultural contexts. Electronic Journal of Research in Educational Psychology, 11, 5 (1), 159-178.

Repetto, E. y Pérez-González, J.C. (2007). Formación en competencias socio-emocionales a través de las prácticas en empresas. Revista Europea de Formación Profesional, 40, 92-112.

Ruiz-Aranda, D., Fernández-Berrocal, P., Cabello, R. y Extremera, N. (2006). Inteligencia emocional percibida y consumo de tabaco y alcohol en adolescentes. Ansiedad y estrés, 12 (2-3), 223-230.

Salovey, P. (2007). Prólogo. En Mestre, J.M. y Fernández-Berrocal, P. (Eds): Manual de Inteligencia emocional, (pp. 17-19). Madrid, Ed. Pirámide.

Senra, M., Pérez-González, J.C. y Manzano, N. (2007). Competencias socioemocionales y alcoholismo en mujeres: revisión y estado actual de la cuestión. Revista Española de Orientación y Psicopedagogía, 18 (1), 73-82.

Vallés, A. (2008). La inteligencia emocional de los padres y de los hijos. Madrid. Pirámide.

Vallés A. y Vallés C. (2000). Inteligencia emocional. Aplicaciones educativas. Madrid: EOS.

Vallés, A. y Vallés, C. (2003). Psicopedagogía de la Inteligencia emocional. Valencia: Promolibro.

Valls, F. (2007). Inteligencia emocional y asesoramiento vocacional y profesional: usos y abusos. Electronic Journal of Research in Educational Psychology, 11, 5 (1), 179-200.

Vila, E. y Pérez-González, J.C. (2007). Madurez para la carrera e inteligencia emocional en alumnado de secundaria: una aproximación correlacional. En F. Etxeberría, L. Sarasola, J.F. Lukas, J. Etxeberría y A. Martxueta (coords.), XIII Congreso Nacional de Modelos de Investigación Educativa (pp. 712-717). Donosita: Erein/AIDIPE (Libro en CD-Rom; ISBN: 978-84-9746-382-9). 